\title{
Type-1 OWA Methodology to Consensus Reaching Processes in Multi-granular Linguistic Contexts
}

\author{
Francisco Mata $^{\mathrm{a}}$, Luis G. Pérez ${ }^{\mathrm{a}}$, Shang-Ming Zhou ${ }^{\mathrm{b}}$, Francisco Chiclana ${ }^{\mathrm{c}}$ \\ ${ }^{a}$ Department of Computer Science, University of Jaén, 23700 Jaén, Spain, fmata@ujaen.es, lgonzaga@ujaen.es \\ ${ }^{b}$ Health Information Research Unit, School of Medicine, Swansea University, SA2 8PP, UK, smzhou@ieee.org \\ ${ }^{c}$ Faculty of Technology, De Montfort University, Leicester, LE1 9BH, UK, chiclana@dmu.ac.uk
}

\begin{abstract}
A crucial step in group decision making (GDM) processes is the aggregation of individual opinions with the aim of achieving a "fair" representation of each individual within the group. In multi-granular linguistic contexts where linguistic term sets with common domain but different granularity and/or semantic are used, the methodology widely applied until now requires, prior to the aggregation step, the application of a unification process. The reason for this unification process is the lack of appropriate aggregation operators for directly aggregating uncertain information represented by means of fuzzy sets. With the recent development of the Type- 1 Ordered Weighted Averaging (T1OWA) operator, which is able to aggregate fuzzy sets, alternative approaches to multi-granular linguistic GDM problems are possible. Unlike consensus models based on unification processes, this paper presents a new T1OWA based consensus methodology that can directly manage linguistic term sets with different cardinality and/or semantic without the need to perform any transformation to unify the information. Furthermore, the linguistic information could be assumed to be balanced or unbalanced in its mathematical representation, and therefore the new T1OWA approach to consensus is more general in its application than previous consensus reaching processes with muti-granular linguistic information. To test the goodness of the new consensus reaching approach, a comparative study between the T1OWA based consensus model and the unification based consensus model is carried out using six randomly generated GDM problems with balanced multi-granular information. When distance between fuzzy sets used in the T1OWA based approach is defined as the corresponding distance between their centroids, a higher final level of consensus is achieved in four out of the six cases although no significant differences were found between both consensus approaches.
\end{abstract}

Keywords: Multi-granular linguistic information, Type-1 OWA operator, Group decision making, Consensus.

\section{Introduction}

Decision making is an inherent activity of human beings. Everyday, human beings, consciously or unconsciously, make decisions about different aspects related to their life. Group decision making (GDM) has proven its usefulness as a decision methodology to address complex decisions in which the participation of experts from different areas may be interesting and even advisable. Moreover, in many of these decision making processes it is common to encounter problems where experts have to assess qualitative aspects that cannot easily be evaluated using precise quantitative assessments. In these cases the use of linguistic assessments seems be more appropriate to express experts' preferences. The fuzzy linguistic approach has 
proven its utility to deal with the imprecision and vagueness associated to qualitative information [1]. In this approach, qualitative aspects are represented by means of linguistic variables whose values are words rather than numbers. Concerning linguistic variables, semantic rules are defined in order to associate to each element of the universe of discourse its meaning. This interpretation of the meaning of a linguistic label is formally captured using the concept of fuzzy set, and therefore linguistic labels can formally be considered and represented as fuzzy subsets of their universe of discourse [1]. Another important aspect to be taken into account in the linguistic approach is the "granularity of uncertainty", i.e. the finest level of distinction among different quantifications of uncertainty as represented by the cardinality of the corresponding linguistic term set [2].

In GDM problems with experts belonging to different areas or with distinct levels of knowledge about the problem, it seems natural to expect that they will express opinions and/or preferences using different sets of linguistic terms and in general with different cardinality (granularity). Consequently, the development of adequate tools to manage and model multi-granular linguistic information becomes very important in the resolution of this type of GDM problem [3-9].

In a multi-granular linguistic context, the aggregation of elements from linguistic term sets of different cardinality and semantics is a challenging issue [10-12]. Different approaches have been proposed in the literature to address this and, among them, one of the most widely used requires a unification process methodology previous to the aggregation operation [6]. The unification process methodology is based on transformation functions with domain each one of the different linguistic term sets and same co-domain, known as the base linguistic term set (BLTS). Although transformation functions operate with the membership functions of the fuzzy sets used to represent the linguistic terms to be aggregated, such functions have been always subject of criticisms because they are not bijective and are subject to possible loss of information. Recently, Zhou et al. [13] proposed the Type-1 Ordered Weighted Average (T1OWA) operator that is able to directly aggregate type-1 fuzzy sets. The T1OWA operator, which is developed via the application of the extension principle to Yager's OWA operator [14], has been successfully proven to aggregate linguistic opinions in human decision making with linguistic weights [15-18]. This operator has the following main characteristics: (i) it allows the direct aggregation of different types of linguistic term sets - balanced or unbalanced sets, triangular or trapezoidal linguistic labels, etc.; (ii) the weighting vector consists of elements that can be crisp and precise numbers or fuzzy ones; (iii) it uses the whole membership function of the fuzzy sets to aggregate in the computation of the fuzzy aggregated value; and (iv) it allows the implementation of the concept of soft majority in the decision process if required. In summary, the use of the T1OWA operator in developing decision making models makes the current unification process superfluous and allows its direct application, i.e. there is no need to modify and/or adapt the model, to a wider range of decision making problems under uncertainty.

GDM problems generally involve situations of conflict among its experts, and therefore it is preferable that the set of experts reach consensus before applying a selection process to derive the decision solution $[8,10,19,20]$. Consensus is defined as the full and unanimous agreement of all the experts, a definition that is inconvenient in practice because it only allows differentiating between two states, namely, the existence and absence of consensus. The chances for reaching such a full agreement are rather low, while it is recognised that most real life situation unanimity is not necessary [21,22]. Thus, one key issue that needs to be addressed in a GDM problem is the evaluation of the level of consensus of the group of experts. Consensus is modelled mathematically via the use of similarity function measuring the concept of proximity 
of information [23]. In the linguistic model, the computation of similarity degrees between experts relies on the use of a distance function between the fuzzy sets representing their linguistic preferences. When the consensus level reaches a threshold value, agreed by the group of experts, the resolution process of the GDM is carried out; otherwise a feedback mechanism is activated, and personalised recommendations generated to support the individual experts, until the threshold level of consensus is achieved. The feedback recommendations will help the experts to identify the preference values that should be considered for changing. The idea of preserving as much as possible the initial experts' preferences [24] has also motived novel methodologies to reach consensus based on linear-programming based approaches that aim at minimising cost under the weighted averaging operator and OWA operators [25].

The aim of this paper is to present a new methodology to consensus reaching processes in multi-granular linguistic contexts based on the T1OWA operator. The new consensus reaching model allows the direct processing of the membership functions of the fuzzy sets modelling the linguistic information and therefore makes the unification process step currently used in developed models unnecessary. Furthermore, because the membership functions are nor required to fulfil extra conditions regarding their balanced or unbalanced distribution within the underlying domain of the variable used to measure preferences, nor they are required to be of the same shape type, the proposed methodology offers a greater degree of flexibility or generality in its application than existing models do. Having said this, a comparative study between the T1OWA based consensus model and the unification based consensus model is included using six randomly generated GDM problems with balanced multi-granular information. As it will be elaborated further later in the paper, when the distance between fuzzy sets in the T1OWA based approach is defined as the corresponding distance between their centroids, a higher final level of consensus is obtained in four out of the six cases studied, although no significant differences are found between both consensus approaches. Arguably, the T1OWA methodology can be used with guarantee in consensus reaching multi-granular linguistic decision making problems.

The rest of the paper is organised as follows. In Section 2 contains a short, but necessary for the set of the paper, review of concepts concerning multi-granular fuzzy linguistic GDM problems, the unification methodology of multi-granular linguistic information and the consensus reaching processes. Section 3 presents the T1OWA operator and its alpha-level fast implementation. Section 4 focuses on the presentation of the new T1OWA methodology to consensus reaching processes in multi-granular linguistic contexts. A comparative study between the new consensus methodology and the consensus methodology based on the unification process of preferences is presented in Section 5. Finally, some conclusions are pointed up in Section 6.

\section{Preliminaries}

To make the paper self-contained, the main concepts that will be used are introduced here.

\subsection{Linguistic variable}

A linguistic variable is formally represented by a 5-tuple $\langle L, T(L), U, S, M\rangle$ [1] where (i) $L$ is the name of the variable; (ii) $T(L)$ is a finite term set of (primary) labels or words (a collection of linguistic values); (iii) $U$ is a universe of discourse or base variable; (iv) $S$ is the syntactic rule which generates the terms in $T(L)$; and (v) $M$ is a semantic rule which associates with each linguistic value $X$ its meaning $M(X): U \rightarrow[0,1]$. Usually, $T(L)$ is denoted as $L$ when there is no risk of confusion. 
The semantic rule, also known as 'compatibility function' [1], associates with each element of the base variable its compatibility with each linguistic value. This interpretation of the meaning of a linguistic label coincides with that of a fuzzy set, and therefore linguistic labels can be considered and formally represented as fuzzy subsets of their base variable. Therefore, the nature of the base variable will dictate the general method to use when manipulating linguistic values. A non numerical base variable makes the definition of the compatibility function 'difficult to be formalized in explicit terms' [1]. As a result, it turns out to be problematic when implemented at present in computer programmes. Thus, it is fair to say that most, if not all, important linguistic decision models in the literature assume that the base variable is a subset of the set of real numbers, and therefore numeric in nature. Indeed, these linguistic decision models usually start associating the linguistic values (labels) to be used with membership functions (triangular, trapezoidal, Gaussian ....) to represent their meanings (see Figure 3).

\subsection{Linguistic decision making problems}

The majority of models developed to solve linguistic GDM problems can be classed as linguistic symbolic computational models based on ordinal scales. Four main proposals can be found in the literature following this ordinal scheme [26]: (i) the linguistic symbolic computational model based on ordinal scales and max-min operators; (ii) the linguistic symbolic computational model based on indexes; (iii) the linguistic symbolic computational model based on continuous term sets, and (iv) the 2-tuple linguistic computational model. The main advantage of these ordinal proposals lies in their simplicity, however they fail to implement appropriately the richness of the linguistic nature that is being modelled in these cases. Indeed, the above models start by assuming a numeric nature of the base variable and membership functions are provided to model the meaning of the linguistic labels used in the problem. However, these membership functions are generally neglected and not fully used, which seems in contradiction with the philosophy of the original linguistic approach in which they are based. In these cases it seems paradoxical that concepts like membership functions, fuzzy sets, vagueness, uncertainty etc. are used in their motivation because they are simply not fully implemented or use in their model architecture. These ordinal models are based on a one-to-one map between the set of linguistic labels and their indexes, which are derived from an underlying ordering the meaning of the linguistic labels obey.

It could be argued, however, that the above mentioned ordinal approaches could be more appropriate for linguistic decision models where the base variable is not numeric in nature, and it could well be that this was the main rationale when developing them. However, they have become so popular within the research community that it seems apparent that they have been adopted by most researchers even when the base variable is numeric in nature. These ordinal approaches do not seem the most appropriate here because their first step consists of replacing the set of linguistic terms, which are supposed to be a set of fuzzy subsets of the set of real numbers, by a set of crisp values before the computation process is carried out, and therefore their expressiveness potential is lost in this way. In these case, Zadeh pointed out the following recommendation to apply when the base variable is numerical in nature: 'linguistic variables can be treated in a reasonably precise fashion by the use of the extension principle for fuzzy sets' [1]. This is the methodology underlying the proposal put forward in this paper.

\subsection{Group decision making with multi-granular linguistic assessments}

GDM problems are classically described as decision situations where, given a set of alternatives $X=\left\{x_{1}, x_{2}, \ldots, x_{n}\right\}(n \geq 2)$, a set of experts $E=\left\{e_{1}, e_{2}, \ldots, e_{m}\right\}(m \geq 2)$ try to achieve a collective solution. Preference relations, also known as pairwise comparison matrices, are a 
popular and powerful method to model experts' preferences in group decision making (GDM) problems. The main advantage of preference relations is that individuals can focus exclusively on two alternatives at a time, which facilitates the expression of their opinions [27-30], making them more accurate than non-pairwise methods [31]. In decision environment pervaded with uncertainty, experts might find comfortable providing their opinions and or preferences using linguistic assessments rather than precise numerical ones $[1,10,26]$. Thus, linguistic preferences relations [8, 32], which are usually modelled as matrices, $P_{e_{i}}=\left(p_{i}^{l k}\right), l, k \in\{1, \ldots, n\}$, will be the focus of this paper. Each element of matrix $p_{i}^{l k}=\mu_{P_{e_{i}}}\left(x_{l}, x_{k}\right)$ represents experts $e_{i}$ linguistic preference of the alternative $x_{l}$ over $x_{k}$, which is formally represented as a fuzzy subset of the unit interval $[0,1]$.

As mentioned before, in GDM we can encounter problems where experts may have different background and level of knowledge about the problem, and consequently linguistic term sets with different cardinality might be used by different experts $[6,33,34]$. In any case, the cardinality or granularity of the linguistic term sets should be small enough so as not to impose useless precision levels but big enough to allow a discrimination of assessments in a limited number of degrees [8]. Summarising, in a multi-granular linguistic GDM context each expert $e_{i}$ will provide preferences using a linguistic term set $S_{i}=\left\{s_{0}^{i}, \ldots, s_{g}^{i}\right\}$, with more two or more linguistic term set having different cardinality.

\subsection{Managing multi-granular linguistic information}

Linguistic preferences expressed on different domains demand specific tools and models to allow the managing of linguistic term sets with different cardinality and/or semantic. A methodology widely used to address GDM problems with multi-granular linguistic preferences is based on a unification process [6], by which experts' linguistic preferences are transformed into a single domain or linguistic term set known as the basic linguistic term set (BLTS), and denoted by $S_{T}$.

Herrera et al. in [6] argue that it seems reasonable to impose a cardinality to $S_{T}$ high enough so that the uncertainty degrees associated to each one of the possible domains used by the experts is maintained. This means that the cardinality of the BLTS has to be as high as possible. In a general multi-granular fuzzy linguistic context, Herrera et al. propose the application of the following rules for the selection of the granularity and semantic of set $S_{T}$ [6]:

1. If there is only one linguistic term set, from the set of different domains to be unified, with maximum granularity, then that set is chosen as the BLTS, $S_{T}$.

2. If there are two or more linguistic term sets with maximum granularity, then the election of $S_{T}$ depends on the semantics associated to them:

(a) If all of them have the same semantics (with different labels), then any one of them can be selected as $S_{T}$.

(b) If two or more of them have different semantics, then $S_{T}$ is defined as a generic linguistic term set with a number of terms greater than the number of terms a person is able to discriminate, which is normally 7 or 9 [35]. There are cases when a BLTS with 15 terms symmetrically distributed has been used [6].

This methodology is further described in an Appendix. Methodologies that do not require the use of a unification process are also available in the literature. A notable example is the methodology developed by Jiang et al. [12], which aims to preserve as much as possible the initial experts' preferences during the decision making process and that is based on the resolution of linear-programming based approaches. 


\subsection{Consensus reaching process}

A consensus reaching process can be defined as an iterative process that consists of several discussion rounds in which experts express their preferences and try to achieve a minimum level of agreement before making a decision [36]. In real-world problems, a human moderator in charged of guiding the experts towards consensual positions that derive in a common and accepted group solution.

A graphical description of a general consensus reaching process is depicted in Fig. 1. As it can be seen, the figure of the moderator has the key role of supervising and coordinating the whole process by providing appropriate advice to the experts in order to bring their positions closer to improve the level of agreement.

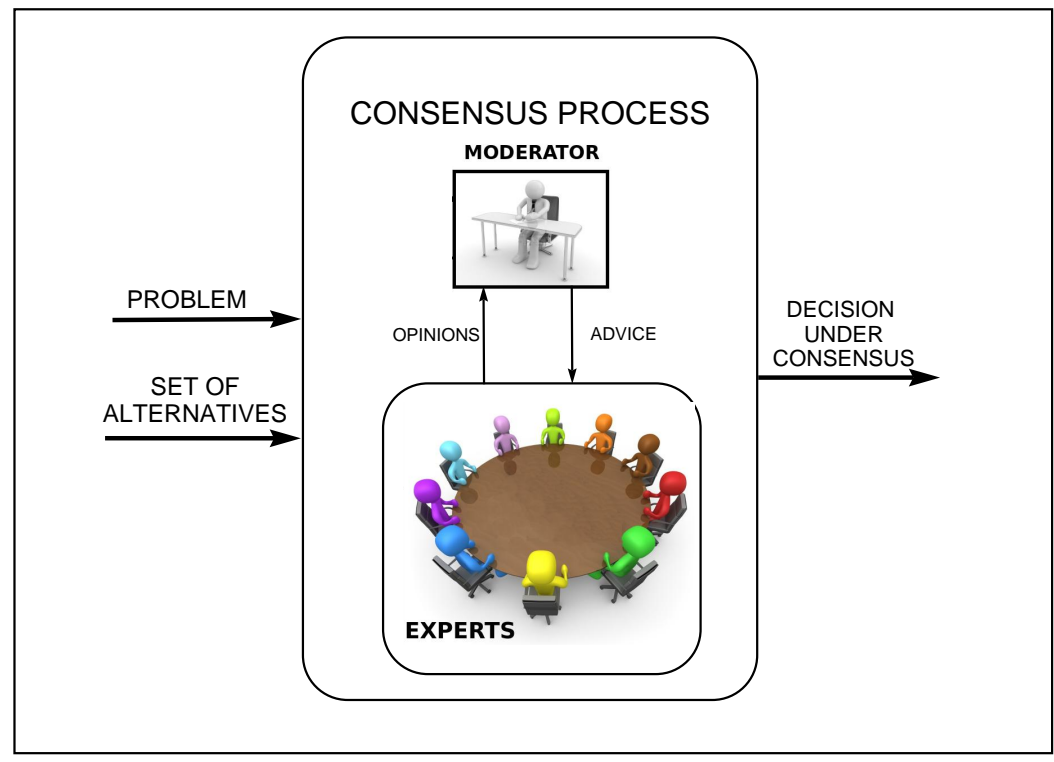

Figure 1: Consensus reaching process

Consensus is usually understood as the full and unanimous agreement of all the experts regarding all the feasible alternatives. However, in practice, this definition is inconvenient because it only allows differentiating between two states: the existence and absence of consensus. Furthermore, on the one hand, the chances for reaching such a full agreement are rather low, and on the other hand, unanimity is not necessary or desirable in most real life situations. A second meaning of the concept of consensus refers to the judgement arrived at by 'most of' those concerned, which has led to the definition and use of a new concept of consensus degree referred to as 'soft' consensus degree [22, 30, 37-44].

\section{Type-1 OWA operator}

Unlike Yager's OWA operator that aggregates crisp values [14], the type-1 OWA operator is able to aggregate type-1 fuzzy sets with uncertain weights, with these uncertain weights being also modelled as type-1 fuzzy sets. As a generalisation of Yager's OWA operator and based on the extension principle, the type-1 OWA operator is defined as follows [13]:

Definition 1 (Type-1 OWA operator (T1OWA)). Given $n$ linguistic weights $\left\{W^{i}\right\}_{i=1}^{n}$ in the form of type-1 fuzzy sets defined on the domain of discourse [0,1], a type-1 OWA operator 
(T1OWA) is a mapping, $\Phi$,

$$
\begin{aligned}
\Phi: \tilde{P}(\mathbb{R}) \times \cdots \tilde{P}(\mathbb{R}) & \longrightarrow \tilde{P}(\mathbb{R}) \\
\left(A^{1}, \cdots, A^{n}\right) & \mapsto Y
\end{aligned}
$$

such that

$$
\begin{aligned}
\mu_{Y}(y)= & \sup \mu_{W^{1}}\left(w_{1}\right) \wedge \cdots \wedge \mu_{W^{n}}\left(w_{n}\right) \wedge \mu_{A^{1}}\left(a_{1}\right) \wedge \cdots \wedge \mu_{A^{n}}\left(a_{n}\right) \\
& \sum_{\substack{k=1 \\
w_{i} \in U, a_{i}}}^{n} \bar{w}_{\sigma(i)}=y
\end{aligned}
$$

where $\bar{w}_{i}=\frac{w_{i}}{\sum_{i=1}^{n} w_{i}} ; \sigma$ is a permutation function such that $a_{\sigma(i)} \geq a_{\sigma(i+1)}, \forall i=1, \cdots, n-1$, and $\tilde{P}(\mathbb{R})$ is the set of fuzzy sets on $\mathbb{R}$.

From Definition 1, a direct approach to performing T1OWA operations was suggested in [13]. However, this approach is computationally expensive, which inevitably curtails further applications of the T1OWA operator to real world decision making. A fast approach to T1OWA operations has been developed based on the $\alpha$-cuts of fuzzy sets [15].

Definition 2 ( $\alpha$-level T1OWA operator ). Given the $n$ linguistic weights $\left\{W^{i}\right\}_{i=1}^{n}$ in the form of type-1 fuzzy sets defined on the domain of discourse $[0,1]$, then for each $\alpha \in[0,1]$, an $\alpha$-level T1OWA operator with $\alpha$-level weight sets $\left\{W_{\alpha}^{i}\right\}_{i=1}^{n}$ to aggregate the $\alpha$-level of type-1 fuzzy sets $\left\{A^{i}\right\}_{i=1}^{n}$ is given as

$$
\Phi_{\alpha}\left(A_{\alpha}^{1}, \cdots, A_{\alpha}^{n}\right)=\left\{\frac{\sum_{i=1}^{n} w_{i} a_{\sigma(i)}}{\sum_{i=1}^{n} w_{i}} \mid w_{i} \in W_{\alpha}^{i}, a_{i} \in A_{\alpha}^{i}, \forall i\right\}
$$

where $W_{\alpha}^{i}=\left\{w \mid \mu_{W_{i}}(w) \geq \alpha\right\}, A_{\alpha}^{i}=\left\{x \mid \mu_{A_{i}}(x) \geq \alpha\right\}$, and $\sigma$ is a permutation function such that $a_{\sigma(i)} \geq a_{\sigma(i+1)}, \forall i=1, \cdots, n-1$.

Using the Representation Theorem of type-1 fuzzy sets, the $\alpha$-level sets $\Phi_{\alpha}\left(A_{\alpha}^{1}, \cdots, A_{\alpha}^{n}\right)$ obtained via Definition 2 can be used to construct the following type-1 fuzzy set on $\mathbb{R}$

$$
G=\underset{0<\alpha \leq 1}{\cup} \alpha \Phi_{\alpha}\left(A_{\alpha}^{1}, \cdots, A_{\alpha}^{n}\right)
$$

with membership function

$$
\mu_{G}(x)=\underset{\alpha: x \in \Phi_{\alpha}\left(A_{\alpha}^{1}, \cdots, A_{\alpha}^{n}\right)_{\alpha}}{\vee} \alpha
$$

Fuzzy sets obtained in (1) and (3) may seem different, however in [15] Zhou et al. proved that both results are equivalent, in what it is known as the Representation Theorem of T1OWA Operators.

Theorem 1 (Representation Theorem of T1OWA Operators). Given the $n$ linguistic weights $\left\{W^{i}\right\}_{i=1}^{n}$ in the form of type-1 fuzzy sets defined on the domain of discourse [0,1], and the type-1 fuzzy sets $A^{1}, \cdots, A^{n}$, then we have that

$$
Y=G
$$

where $Y$ is the aggregation result defined in (1) and $G$ is the result defined in (3). 
Therefore, an effective and practical way of carrying out T1OWA operations consist in the decomposition of the T1OWA aggregation into the $\alpha$-level T1OWA operations and then the reconstruction of the output via the Representation Theorem 1.

When the linguistic weights and the aggregated sets are fuzzy number, the output of the $\alpha$-level T1OWA operator is a closed interval [15]:

Theorem 2. Let $\left\{W^{i}\right\}_{i=1}^{n}$ be fuzzy numbers on $[0,1]$ and $\left\{A^{i}\right\}_{i=1}^{n}$ be fuzzy numbers on $\mathbb{R}$. Then for each $\alpha \in U, \Phi_{\alpha}\left(A_{\alpha}^{1}, \cdots, A_{\alpha}^{n}\right)$ is a closed interval.

Based on this result, the computation of the T1OWA output according to (3), $G$, reduces to compute the left end-points and right end-points of the intervals $\Phi_{\alpha}\left(A_{\alpha}^{1}, \cdots, A_{\alpha}^{n}\right)$ :

$$
\Phi_{\alpha}\left(A_{\alpha}^{1}, \cdots, A_{\alpha}^{n}\right)_{-} \text {and } \Phi_{\alpha}\left(A_{\alpha}^{1}, \cdots, A_{\alpha}^{n}\right)_{+},
$$

where $A_{\alpha}^{i}=\left[A_{\alpha-}^{i}, A_{\alpha+}^{i}\right], W_{\alpha}^{i}=\left[W_{\alpha-}^{i}, W_{\alpha+}^{i}\right]$. For the left end-points, we have

$$
\begin{aligned}
\Phi_{\alpha}\left(A_{\alpha}^{1}, \cdots, A_{\alpha}^{n}\right)_{-}= & \min W_{\alpha-}^{i} \leq w_{i} \leq W_{\alpha+}^{i} \sum_{i=1}^{n} w_{i} a_{\sigma(i)} / \sum_{i=1}^{n} w_{i} \\
& A_{\alpha-}^{i} \leq a_{i} \leq A_{\alpha+}^{i}
\end{aligned}
$$

For the right end-points, we have

$$
\begin{aligned}
\Phi_{\alpha}\left(A_{\alpha}^{1}, \cdots, A_{\alpha}^{n}\right)_{+}= & \max W_{\alpha-}^{i} \leq w_{i} \leq W_{\alpha+}^{i} \sum_{i=1}^{n} w_{i} a_{\sigma(i)} / \sum_{i=1}^{n} w_{i} \\
& A_{\alpha-}^{i} \leq a_{i} \leq A_{\alpha+}^{i}
\end{aligned}
$$

It can be seen that (5) and (6) are programming problems. Solutions to these problems, so that the T1OWA aggregation operation can be performed efficiently, are available from [15].

\section{Consensus reaching process based on T1OWA operator}

In the multi-granular context, the different theoretical models proposed in the literature $[8,30,38]$ address the consensus reaching process, in which transformation functions were used to unify the multi-granular linguistic information. As pointed our earlier, a drawback of such unification process is that the consensus reaching process loses information because the transformations functions are not bijective. These proposed models did not address the issues of direct manipulation of different elements belonging to linguistic sets of different granularity, specially in the necessary aggregation step of GDM problems, because there were no mathematical tools available at that moment. Interestingly, the introduction of the T1OWA operator provides such a needed tool for the direct manipulating linguistic sets with different granularity in consensus decision making. Unlike previous models [8,38], the proposed consensus model makes use of the T1OWA operator to compute the collective preference, and therefore the whole membership functions of the fuzzy sets that represent the experts' linguistic preferences are taken into account. The two main advantage with respect to previous models are: (1) it allows the direct aggregation of different types of linguistic term sets - balanced or unbalanced sets, triangular or trapezoidal linguistic labels, etc. and therefore the unification process is not just necessary; and (2) there is no loss of information in the aggregation process.

A general sketch of the consensus process proposed is depicted in Figure 2. The model has a set of multi-granular linguistic preferences as input, with a final output being a set of recommendations to the group of experts to increase the level of agreement when this is below a threshold value for the next consensus round. Specifically our proposed model includes the following steps: 
1. Computation of the consensus degree: (1.1) Similarity degrees; and (2.2) Consensus matrix.

\section{Consensus control.}

3. Feedback Mechanism: (3.1) Computation of the collective linguistic preference relation; (3.2) Identification of the preference values to change; and (3.3) Generation of advice.

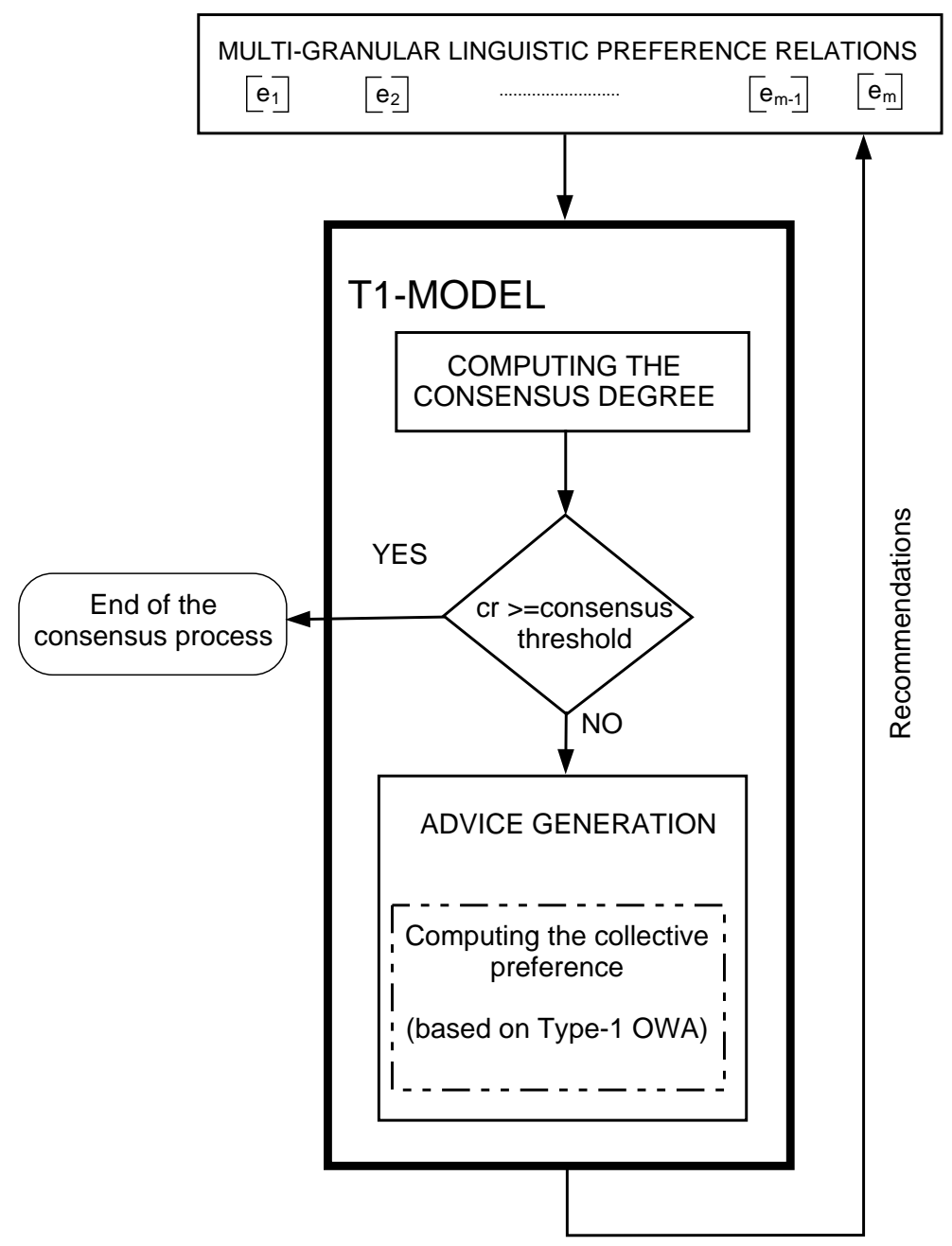

Figure 2: T1OWA based consensus model

These steps will be presented in more detail in following subsections. A step-by-step example to illustrate the computation processes involved in each step is also provided. This example is taken from [8] where a unification methodology to consensus was applied to.

Example 1. An investment company wants to invest a sum of money in the best industrial sector among four possible alternatives: car industry $\left(x_{1}\right)$, food company $\left(x_{2}\right)$, computer factory $\left(x_{3}\right)$ and arms industry $\left(x_{4}\right)$. Before making a decision a minimum level of agreement among four experts, $\left\{e_{1}, \ldots, e_{4}\right\}$, is required. These experts belong to different departments within the company (risk analysis, growth analysis, social-political analysis and environmental impact analysis department) and they use linguistic term sets with different granularity and semantics. 
Two key parameters in any consensus reaching process are the consensus threshold, i.e. the minimum level of agreement that experts want to achieve, and the maximum number of consensus rounds that they are willing to carry out in order to achieve it. Both parameters have to be fixed in advance and their values depend on the type of problem. In this example, these parameters are set to be 0.75 and 10 , respectively.

Experts provide preferences using one of the possible three linguistic label sets $A, B, C$ with elements modelled using triangular fuzzy numbers (TFNs) in the numeric domain $[0,1]$, as represented in Figure 3.
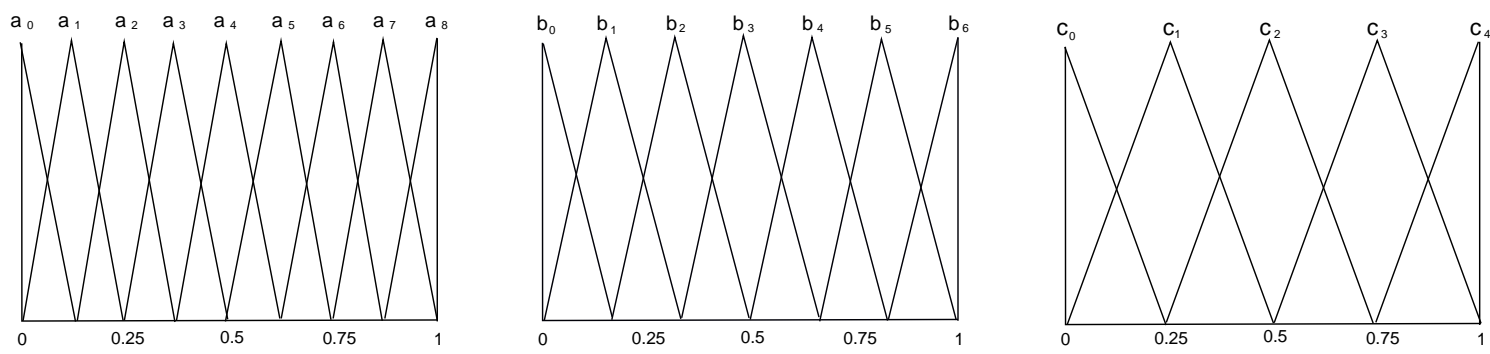

Figure 3: Linguistic label sets $A, B$ and $C$ (from left to right)

A triangular fuzzy number (TFN) membership function $\mu:[0,1] \rightarrow[0,1]$ is:

$$
\mu_{\widetilde{A}}(x)= \begin{cases}0, & 0 \leq x<a \\ \frac{x-a}{b-a}, & a<x \leq b \\ \frac{c-x}{c-b}, & b \leq x<c \\ 0, & c<x \leq 1\end{cases}
$$

A TFN can shortly represented in its parametric form $(a, b, c)$, with $a$ and $c$ known as the lower and upper bounds, respectively, while $b$ is known as its modal value. When the TFN is symmetrical, i.e. when $b=(a+c) / 2$, then $b$ is also its centroid. The parametric representation of the above three linguistic label sets $A, B, C$ using TFNs is given in Table 1.

\begin{tabular}{|l|l|l|}
\hline Label set A & Label set B & Label set C \\
\hline \hline$a_{0}=(0,0,0.12)$ & $b_{0}=(0,0,0.16)$ & $c_{0}=(0,0,0.25)$ \\
$a_{1}=(0,0.12,0.25)$ & $b_{1}=(0,0.16,0.33)$ & $c_{1}=(0,0.25,0.5)$ \\
$a_{2}=(0.12,0.25,0.37)$ & $b_{2}=(0.16,0.33,0.5)$ & $c_{2}=(0.25,0.5,0.75)$ \\
$a_{3}=(0.25,0.37,0.5)$ & $b_{3}=(0.33,0.5,0.66)$ & $c_{3}=(0.5,0.75,1)$ \\
$a_{4}=(0.37,0.5,0.62)$ & $b_{4}=(0.5,0.66,0.83)$ & $c_{4}=(0.75,1,1)$ \\
$a_{5}=(0.5,0.62,0.75)$ & $b_{5}=(0.66,0.83,1)$ & \\
$a_{6}=(0.62,0.75,0.87)$ & $b_{6}=(0.83,1,1)$ & \\
$a_{7}=(0.75,0.87,1)$ & & \\
$a_{8}=(0.87,1,1)$ & & \\
\hline
\end{tabular}

Table 1: TFN parametric representation of linguistic label sets $A, B$ and $C$

Example 2 (Example 1 continuation). Experts $e_{1}$ and $e_{2}$ provide linguistic assessment using set $C$, while expert $e_{3}$ uses set $A$ and expert $e_{4}$ set $B$. Their initial linguistic preference relations being: 


$$
\begin{aligned}
\mathbf{P}_{\mathbf{e}_{1}} & =\left(\begin{array}{cccc}
- & c_{0} & c_{0} & c_{2} \\
c_{4} & - & c_{3} & c_{4} \\
c_{3} & c_{0} & - & c_{1} \\
c_{2} & c_{1} & c_{3} & -
\end{array}\right) \mathbf{P}_{\mathbf{e}_{2}}=\left(\begin{array}{cccc}
- & c_{2} & c_{0} & c_{4} \\
c_{1} & - & c_{1} & c_{1} \\
c_{3} & c_{3} & - & c_{1} \\
c_{0} & c_{4} & c_{3} & -
\end{array}\right) \\
\mathbf{P}_{\mathbf{e}_{3}} & =\left(\begin{array}{cccc}
- & a_{1} & a_{4} & a_{3} \\
a_{5} & - & a_{8} & a_{4} \\
a_{4} & a_{1} & - & a_{2} \\
a_{5} & a_{5} & a_{7} & -
\end{array}\right) \mathbf{P}_{\mathbf{e}_{4}}=\left(\begin{array}{cccc}
- & b_{0} & b_{4} & b_{5} \\
b_{6} & - & b_{1} & b_{6} \\
b_{3} & b_{4} & - & b_{2} \\
b_{0} & b_{1} & b_{4} & -
\end{array}\right)
\end{aligned}
$$

\subsection{Computing the consensus degree}

It was mentioned before that consensus is a measure of agreement, and thus in the context of GDM it will represent the level of agreement among the experts. Mathematically, consensus can be defined using similarity functions measuring the concept of proximity of information [23]. In the linguistic model, the computation of similarity degrees between experts relies on the use of a distance function between the fuzzy sets representing their linguistic preferences. This is elaborated in the following subsections.

\subsubsection{Similarity degress}

In the following, we provide the formal definition of distance and similarity functions as given in [45]:

Definition 3 (Distance). Let $A$ be a set. A function $d: A \times A \longrightarrow \mathbb{R}$ is called a distance (or disimilarity) on $A$ if, for all $x, y \in A$, there holds

1. $d(x, y) \geq 0$ (non-negativity)

2. $d(x, y)=d(y, x)$ (symmetry)

3. $d(x, x)=0$ (reflexivity)

Definition 4 (Similarity). Let $A$ be a set. A function $s: A \times A \longrightarrow \mathbb{R}$ is called a similarity on $A$ if $s$ is non-negative, symmetric, and if $s(x, y) \leq s(x, x)$ holds for all $x, y \in A$, with equality if and only if $x=y$.

The main transforms between a distance $d$ and a similarity $s$ bounded by 1 are [45]:

$$
d=1-s ; d=\frac{1-s}{s} ; d=\sqrt{1-s} ; d=\sqrt{2 \cdot\left(1-s^{2}\right)} ; d=\arccos s ; d=-\ln s
$$

In this paper, we use the first transform to go from a distance function to a similarity function.

There are two main approaches to compare two fuzzy sets $A_{1}$ and $A_{2}$ using a metric, $d\left(A_{1}, A_{2}\right)$. The first approach, based on the application of the extension principle to a numeric distance such as the Hamming or Euclidean distances [46-48], to extend their application to the case of fuzzy sets. The output of such metric is a fuzzy set. However, it is well known that the set of fuzzy sets (numbers) is not totally ordered [49], and therefore alternative approaches might be needed if this type of ordering is required. A widely used approach in this case is to convert the fuzzy sets into a representative crisp value, and perform the comparison on these representative values [50,51]. This is the approach implemented in this consensus model, with the centroid being the representative element of the corresponding linguistic label used to compute similarity degrees. 
Definition 5. The centroid of a type-1 fuzzy set $A$ in a continuous domain X is calculated as,

$$
c v_{A}=\frac{\int_{x} x \cdot \mu_{A}(x) d x}{\int_{x} \mu_{A}\left(x_{i}\right)} .
$$

The centroid of linguistic preference $p_{i}^{l k}$ provided by expert $e_{i}$ when comparing the ordered pair of alternatives $\left(x_{l}, x_{k}\right)$ will be denoted by $c v e_{i}^{l k}$. Thus the first step to compute similarity degrees between experts is to derive their corresponding matrix of centroids.

Example 3 (Example 1 end). We only show here the matrix of centroids of expert $e_{1}$ :

$$
C V e_{1}=\left(\begin{array}{cccc}
- & 0.0833 & 0.0833 & 0.5 \\
0.9167 & - & 0.75 & 0.9167 \\
0.75 & 0.0833 & - & 0.25 \\
0.5 & 0.25 & 0.75 & -
\end{array}\right)
$$

Note 1. Unlike previous models $[8,38]$, the elements of the matrix of centroids are now more meaningful and understandable. For example, the linguistic preference value $p_{1}^{14}=c_{2}$ given by $e_{1}$ represents a central assessment of indifference within the linguistic set $C$, and therefore its associated representative value should be the equivalent in the numeric preference domain, i.e. 0.5 , as it is the case with its centroid value, $c v e_{1}^{14}=0.5$.

Definition 6 (Similarity degree between fuzzy sets). Given two fuzzy sets, $A_{1}$ and $A_{2}$, their similarity $s\left(A_{1}, A_{2}\right)$ is:

$$
s\left(A_{1}, A_{2}\right)=1-d\left(c v_{A_{1}}, c v_{A_{2}}\right)
$$

In the particular case of TFNs $A_{1}=\left(a_{1}, b_{1}, c_{1}\right)$ and $A_{2}=\left(a_{2}, b_{2}, c_{2}\right)$ we have $c v_{A_{1}}=\left(a_{1}+b_{1}+c_{1}\right) / 3$ and $c v_{A_{2}}=\left(a_{2}+b_{2}+c_{2}\right) / 3$. Using the Hamming distance

$$
s\left(A_{1}, A_{2}\right)=1-\left|\frac{\left(a_{1}-a_{2}\right)+\left(b_{1}-b_{2}\right)+\left(c_{1}-c_{2}\right)}{3}\right| .
$$

An alternative expression for the distance between TFNs is [52]:

$$
d^{\prime}\left(A_{1}, A_{2}\right)=\frac{\left|a_{1}-a_{2}\right|+\left|b_{1}-b_{2}\right|+\left|c_{1}-c_{2}\right|}{3} .
$$

Expression (10) will be used in this paper.

For each pair of experts $e_{i}, e_{j}(i<j)$, a similarity matrix, $S M_{i j}=\left(s m_{i j}^{l k}\right)$, is obtained

$$
s m_{i j}^{l k}=s\left(p_{i}^{l k}, p_{j}^{l k}\right)
$$

where $s m_{i j}^{l k}$ represent the similarity degree between the preferences of the experts $e_{i}$ and $e_{j}$ on the ordered pair of alternatives $\left(x_{l}, x_{k}\right)$. The closer $s m_{i j}^{l k}$ to 1 the more similar $p_{i}^{l k}$ and $p_{j}^{l k}$ are considered, while the closer $s m_{i j}^{l k}$ to 0 the more distant $p_{i}^{l k}$ and $p_{j}^{l k}$ are considered.

Note 2. By definition, we have $s m_{i j}^{l k}=s m_{j i}^{l k}$ and therefore we can impose the constraint $i<j$ when computing similarity matrices. 


\subsubsection{Consensus matrix}

As it was mentioned before, consensus within a group of experts will measure the level of agreement of all the experts in the group on the question to solve. This definition implicitly implies that consensus can be mathematically modelled by fusing the similarity degrees previously defined. Thus, the aggregated value of all the similarity degrees on a particular ordered pair of alternatives will represent the degree of consensus of the group of experts on that particular pair of alternatives. The matrix obtained in such a way will therefore be referred to as the consensus matrix, $C M=\left(\mathrm{cm}^{l k}\right)$, i.e.

$$
\forall l, k=1, \ldots, n: c m^{l k}=\phi\left(s m_{i j}^{l k}\right) ; i, j=1, \ldots, m \wedge i<j .
$$

The operator $\phi$ represents an appropriate aggregation operator that will change depending on the problem to be solved or the constraints imposed to the problem. For example, if a solution of consensus is to be achieved when 'most of' the expert agree on it the aggregation operator could well be Yager's OWA operator guided by the linguistic quantifier representing the concept 'most of'. Because, one of our objectives in this papers is to compare the new consensus methodology here presented with the unification based consensus model presented in [8], we will be using the simple average as the aggregation operator $\phi$.

Consensus can be measured at the three different levels of the relation: pair of alternatives, alternatives and relation [53]:

Level 1. Consensus on pairs of alternatives to measure the degree of consensus of the group of experts on the ordered pair of alternatives:

$$
c p^{l k}=c m^{l k}, \forall l, k=1, \ldots, n \wedge l \neq k .
$$

Level 2. Consensus on alternatives to measure the degree of consensus of the group of experts on the alternatives:

$$
c a^{l}=\frac{\sum_{k=1, l \neq k}^{n} c p^{l k}}{(n-1)} .
$$

Level 3. Consensus on the relation or global consensus of the group of experts

$$
c r=\frac{\sum_{l=1}^{n} c a^{l}}{n} .
$$

Example 4 (Example 1 continuation). The degree of consensus at the three different levels are:

Level 1. Consensus on pairs of alternatives: $C M=\left(\begin{array}{cccc}- & 0.7706 & 0.6422 & 0.6717 \\ 0.6039 & - & 0.5183 & 0.5839 \\ 0.8333 & 0.5757 & - & 0.9600 \\ 0.6472 & 0.5600 & 0.8950 & -\end{array}\right)$.

Level 2. Consensus on alternatives: $c a^{1}=0.6948 ; c a^{2}=0.5687 ; c a^{3}=0.7900 ; c a^{4}=0.7007$.

Level 3. Global consensus: $c r=0.6886$. 


\subsection{Consensus control}

The global consensus degree $c r$ obtained in the previous phase is compared against a consensus threshold, $\lambda$, agreed by the set of expert previous to the application of the consensus process. If $\mathrm{cr}$ is greater or equal than this consensus threshold then the consensus reaching process is considered successful and hence it should end. Otherwise, the experts need to discuss and attempt to bring their opinions closer for their consensus degree to increase and achieve the threshold value set. In the following we present a feedback mechanism to generate advice rules to support the experts in achieving this goal. Note that the model uses a parameter, Maxrounds, which represents the maximum number of rounds allowed before stopping the consensus process. In this way the model guarantees the end of the process. Both parameters, $\lambda$ and Maxrounds, must be fixed in advance before starting the consensus process.

\subsection{Feedback mechanism}

When $c r<\lambda$ a feedback mechanism is activated to generate personalised advice to some or all of the experts on a number of preference values that are identified as contribution low to consensus, i.e. those preference values whose similarity to the corresponding collective preference value are below the consensus threshold value. To do this, the following there steps are carried out: Computation of the collective linguistic preference relation, Identification of the preference values that should be changed and Generation of advice.

\subsubsection{Computation of the collective linguistic preference relation}

The collective linguistic preference relation, $\mathbf{P}_{\mathbf{c o l}}$, is calculated by aggregating all experts' preference relations $\left\{\mathbf{P}_{\mathbf{e}_{\mathbf{1}}}, \ldots, \mathbf{P}_{\mathbf{e}_{\mathbf{m}}}\right\}$ at the level of pairs of alternatives. This aggregation is carried out using the T1OWA operator:

$$
\mathbf{P}_{\text {col }}=\Phi\left(\mathbf{P}_{\mathbf{e}_{1}}, \ldots, \mathbf{P}_{\mathbf{e}_{\mathbf{m}}}\right) .
$$

In this way, the model can aggregate directly multi-granular linguistic labels represented by fuzzy sets without loss of information, being this one of the key aspect of the model.

The similarity between the collective linguistic preference values and the corresponding individual ones will be used to identify the preferences values to be changed. Thus, the corresponding matrix of centroid, $C V_{c o l}$, associated to the collective linguistic preference relation is also computed here.

Example 5 (Example 1 continuation). Figure 4 shows the individual linguistic preferences provided by each one of the experts when comparing the ordered pair of alternatives $\left(x_{1}, x_{2}\right)$ (solid lines) and the aggregation result (dashed line) using the T1OWA implementation of the average operator.

The collective matrix of centroids is:

$$
C V_{\text {col }}=\left(\begin{array}{cccc}
- & 0.1922 & 0.3341 & 0.6537 \\
0.6813 & - & 0.5294 & 0.6488 \\
0.6238 & 0.4061 & - & 0.2695 \\
0.3175 & 0.4877 & 0.7588 & -
\end{array}\right)
$$

Note 3. The visualisation of expert's opinions with respect to the collective one as shown in Figure 4 constitutes a powerful tool to support the experts in understanding their standing with respect to, on the one hand, the group and, on the either hand, another expert within the group he/she might be interested to follow or analyse. It can be easily deducted from Figure 4 that expert $e_{2}$ is the furthers of all with respect to the collective linguistic preference, while expert $e_{3}$ linguistic preference value is very similar to the collective one. 


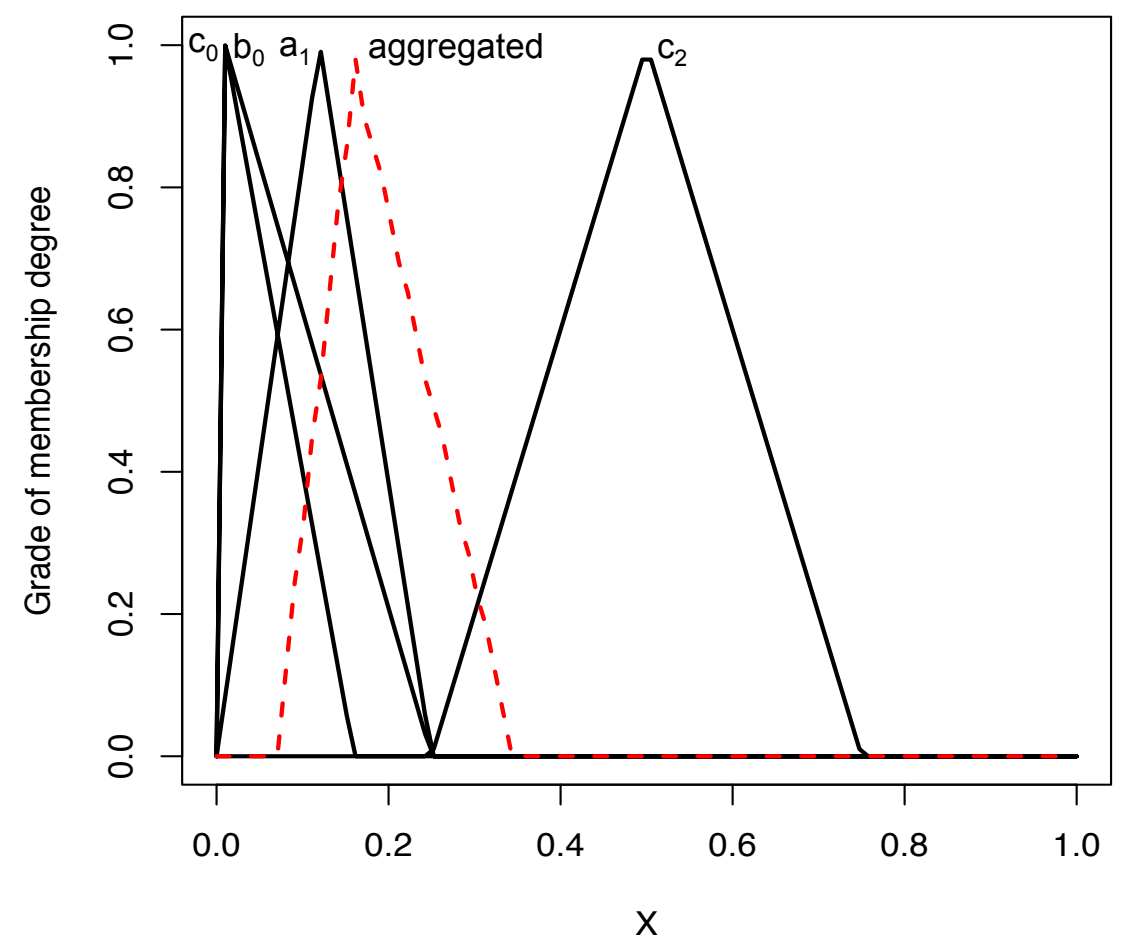

Figure 4: T1OWA aggregation: solid lines: individual linguistic preferences represented as TFNs ; dashed line: collective linguistic aggregation result, also a TFN

\subsubsection{Identification of the preference values to change}

In order to increase the global consensus degree, $c_{r}$, the first step is to identify those alternatives that contribute less to consensus, i.e. the alternatives $\left(x_{l}\right)$ with a consensus degree, $c a^{l}$, lower than the global consensus degree. Next, for each identified alternative, the pairs of alternatives with a consensus value, $c p^{l k}$, below the global consensus degree are proposed to be changed because these are the values that contribute less to consensus. However, not all experts will require to modified the identified preference values but those with a proximity value with respect to the collective preference at these pairs of alternatives lower than the average proximity value for the whole group.

Therefore the steps to follow are:

Computing the proximity matrix Using the distance function (11), a proximity matrix is computed for each expert, $P M_{i}=\left(p m_{i}^{l k}\right)$ :

$$
p p_{i}^{l k}=1-\left|c v e_{i}^{l k}-c v_{c o l}^{l k}\right|
$$

where $p p_{i}^{l k}$ represents the proximity between the preference of expert $e_{i}$ and the collective one on the pair of alternatives $\left(x_{l}, x_{k}\right)$.

Identification of alternatives The set of alternatives with a consensus degree lower than the threshold value is identified:

$$
I A=\left\{l \in\{1, \ldots, n\} \mid c a^{l}<c r\right\} .
$$


Identification of preferences The set of preference values to consider for change in the next consensus round is:

$$
I P=\left\{(l, k) \in\{1, \ldots, n\}^{2} \mid l \in A \wedge c p^{l k}<c r\right\}
$$

Preference values to change The set of preference values to change and the experts that will be requested to change them is:

$$
P C=\left\{(i, l, k) \in\{1, \ldots, m\} \times\{1, \ldots, n\}^{2} \mid \max \left\{c a^{l}, c p^{l k}\right\}<c r \wedge p p_{i}^{l k}<\frac{\sum_{j=1}^{m} p p_{i}^{l k}}{m}\right\} .
$$

Note 4. In (20), a general aggregation operator $\theta$ could be used to compute the average proximity of the group at the level of pairs of alternatives, $\overline{p p}^{l k}=\theta\left(p p_{1}^{l k}, \ldots, p p_{m}^{l k}\right)$. Thus, the general definition of the set $P C$ would be:

$$
P C=\left\{(i, l, k) \in\{1, \ldots, m\} \times\{1, \ldots, n\}^{2} \mid \max \left\{c a^{l}, c p^{l k}\right\}<c r \wedge p p_{i}^{l k}<\overline{p p}^{l k}\right\} .
$$

Example 6 (Example 1 continuation). Applying the rules given above we have:

$$
\begin{aligned}
& I A=\{2\} . \\
& I P=\{(2,1),(2,3),(2,4)\} . \\
& P C=\{(2,2,1),(2,2,4),(2,2,3),(4,2,1),(4,2,3),(4,2,4)\} .
\end{aligned}
$$

\subsubsection{Generation of advice}

The experts previously identify to change some of their preferences will receive personalised recommendations of the direction of the change expected to be done if consensus is to be increased. For all $(i, l, k) \in P C$, the following two direction rules are proposed:

DR.1. If $c v e_{i}^{l k}-c v_{c o l}^{l k}<0$, then expert $e_{i}$ should increase the linguistic assessment associated to the pair of alternatives $\left(x_{l}, x_{k}\right)$.

DR.2. If $c v e_{i}^{l k}-c v_{c o l}^{l k}>0$, then expert $e_{i}$ should decrease the linguistic assessment associated to the pair of alternatives $\left(x_{l}, x_{k}\right)$.

Note 5. The case $c v e_{i}^{l k}-c v_{c o l}^{l k}=0$ is not possible to happen at this stage. Indeed, if we have $c v e_{i}^{l k}-c v_{c o l}^{l k}=0$ then by (17) it would be $p p_{i}^{l k}=1$, i.e. the proximity between the preference value of expert $e_{i}$ and the collective preference value on the pair of alternatives $\left(x_{l}, x_{k}\right)$ would be maximum and, consequently, we would have that $(i, l, k) \notin P C$.

Note 6. It is up to the experts to decide on the magnitude of the change to implement as the rules only indicate the direction of the change. Consequently, the model here presented cannot be categorised as an automatic consensus reaching process.

Example 7 (Example 1 end). Assuming that the experts implement the recommended changes by choosing as new linguistic preferences the linguistic label that is just before or the following one in the ordinal scale defined by their meaning and/or the corresponding centroids of their associated TFNs, we summarise the main parameters of whole consensus process in Table 2. 


\begin{tabular}{|l|c|c|}
\hline Round number & Consensus degree & Number of changes \\
\hline \hline First round & 0.6885 & 7 \\
\hline Second round & 0.7283 & 7 \\
\hline Third round & 0.7648 & End \\
\hline
\end{tabular}

Table 2: Consensus process results

\section{Comparative study: T1OWA model vs. Unification model}

The output of the transformation function, $\tau_{S S_{T}}$, given in (23) when applied to linguistic preference values, $p_{i}^{l k}$, (with TFNs as membership functions) is a fuzzy set on the BLTS, $S_{T}=$ $\left\{c_{0}, \ldots, c_{g}\right\}, \tilde{p}_{i}^{l k}=\tau_{S_{i} S_{T}}\left(p_{i}^{l k}\right)=\left(\alpha_{i 0}^{l k}, \ldots, \alpha_{i g}^{l k}\right)$. Formally, this is a discrete type-2 fuzzy sets $[54,55]$. However, in [6] these type-2 fuzzy set were transformed into discrete type-1 fuzzy sets by associating to each element (linguistic label), $c_{h}$, of the BLTS set its subindex, $h$, in order to carry out the next step of the unification based consensus model, i.e. the computation of the centroid of the unified linguistic preferences using the discrete version of the Equation (8):

$$
c v e_{i}^{l k}=c v\left(\tilde{p}_{i}^{l k}\right)=\frac{\sum_{h=0}^{g} h \cdot \alpha_{i h}^{l k}}{\sum_{h=0}^{g} \alpha_{i h}^{l k}} .
$$

The T1OWA based consensus process and the unification based consensus process were applied to six randomly generated GDM problem with multi-granular linguistic information for comparative purposes. The following constraints were imposed:

(i) Number of experts and alternatives were kept low as in Example 1.

(ii) The same three sets $A, B$ and $C$ of symmetric and balanced linguistic terms of Example 1 were used.

(iii) A consensus threshold $\gamma=0.75$ and Max_rounds $=10$ were fixed in advance.

Both consensus models were implemented and executed with the mathematical software MATLAB. The T1OWA operations were carried out using the R-software environment [56]. Figure 5 depicts the evolution of the global consensus degree for each model during the different consensus rounds. The T1OWA based consensus model achieves a final global consensus higher than the unification based model in four out of the six cases. Figure 6 summarises the changes suggested and implemented in each consensus round as well as the aggregated number of changes for both models. It can be seen that the unification based model achieves a higher final consensus degree than the T1OWA based model when its aggregated number of changes is higher, otherwise the T1OWA based model achieves a higher final degree of consensus.

The above comparison cannot be considered a rigorous technique because values that differ might not be significantly different from a statistical point of view. Greater rigour can be achieved through statistical testing. The Wilcoxon Signed Rank Test [57] was applied to the final global consensus achieved to ascertain whether or not there was a significant difference between both consensus model using the centroid values to define consensus and proximity degrees. Thus, the hypothesis tested was:

$H_{0}$ : The T1OWA based consensus model and the Unification based consensus model do not produce significantly different global consensus degrees. 

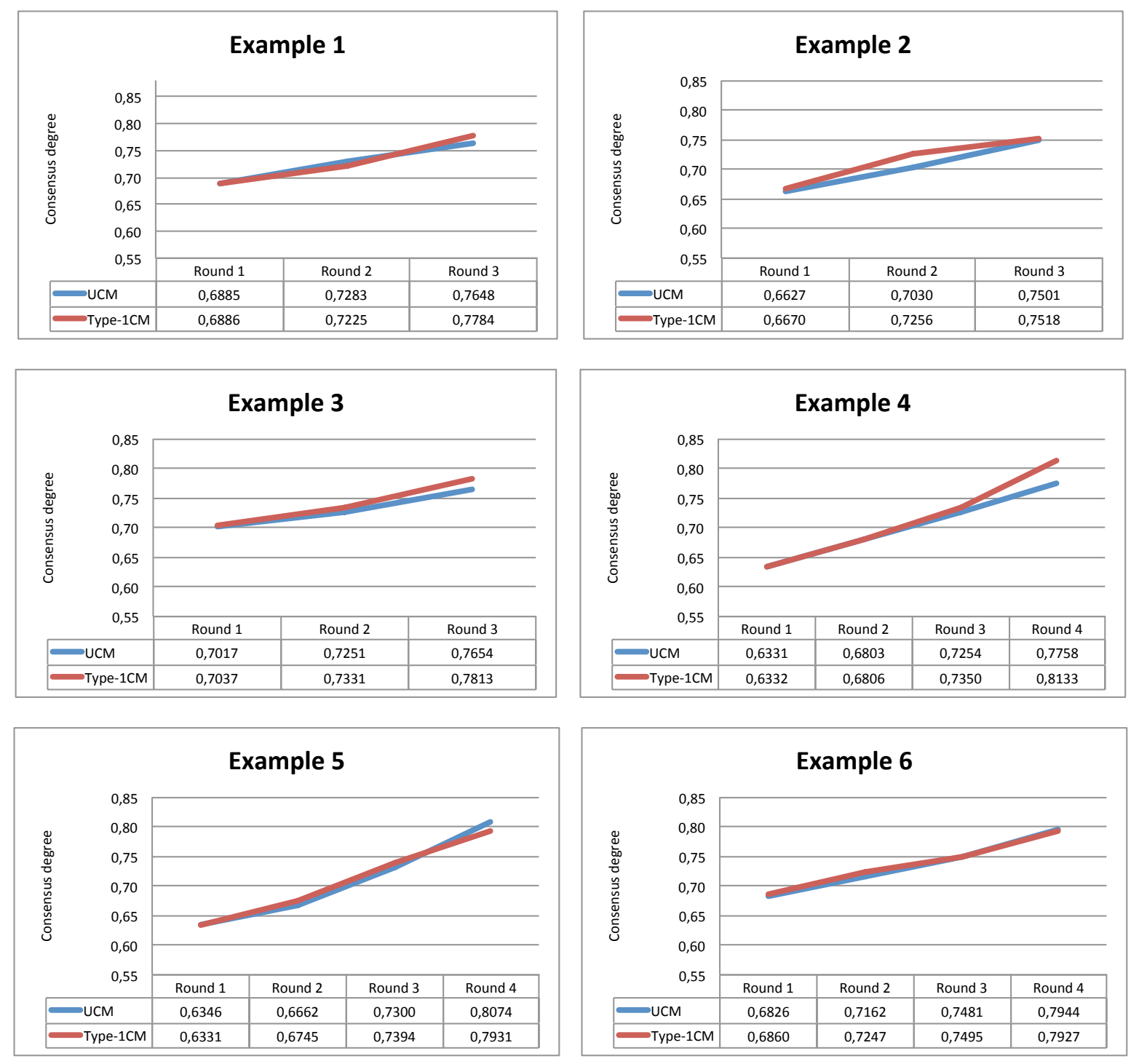

Figure 5: T1OWA model v Unification model: Comparison of the evolution of the consensus degree

Table 3 shows the computation necessary to apply the one-sided Wilcoxon Signed Rank Test, i.e. the alternative hypothesis was:

$H_{1}$ : The T1OWA based consensus model results in global consensus degrees significantly higher than the Unification based consensus model.

From Table 3 we compute the statistic: $T_{-}=5$, i.e. the sum of absolute ranks assigned to negative differences. We assume that a test $p$-value under the null hypothesis lower than or equal to $0.05(\alpha)$ will be considered as significantly different; we refer to it as the test being significant and therefore we conclude that the null hypothesis tested is to be rejected. Otherwise, we will fail to reject the null hypothesis. In terms of the value $T_{-}$, the null hypothesis tested is to be rejected in favour of the alternative one when $T_{-}$is lower than or equal to the critical value. For a sample size of 6 , the critical value is 2 and therefore we conclude that we fail to reject the null hypothesis. We conclude that both consensus models do not produce significantly different global consensus degrees. 


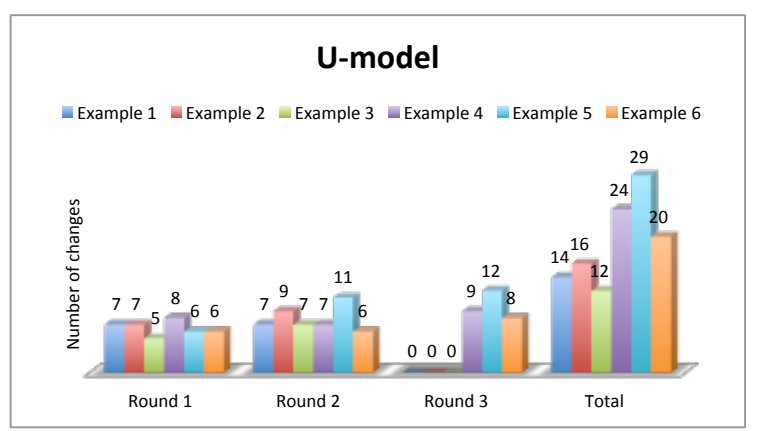

(a) U-model

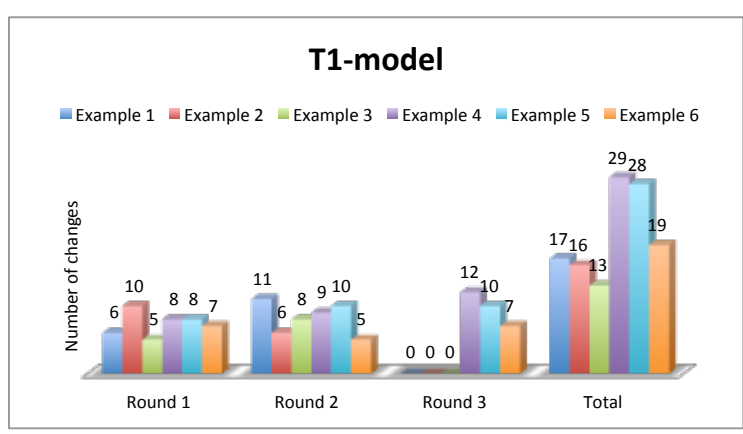

(b) T1-model

Figure 6: Comparison of number of changes

\begin{tabular}{|c|c|c|c|c|c|c|}
\hline TEST SET & T1-model & U-model & Differences & Absolute Value & Rank & Signed Rank \\
\hline \hline Example 1 & 0.7784 & 0.7648 & 0.0136 & 0.0136 & 3 & 3 \\
\hline Example 2 & 0.7518 & 0.7501 & 0.0017 & 0.0017 & 2 & 2 \\
\hline Example 3 & 0.7813 & 0.7654 & 0.0159 & 0.0159 & 5 & 5 \\
\hline Example 4 & 0.8133 & 0.7758 & 0.0375 & 0.0375 & 6 & 6 \\
\hline Example 5 & 0.7931 & 0.8074 & -0.0143 & 0.0143 & 4 & -4 \\
\hline Example 6 & 0.7931 & 0.7944 & -0.0013 & 0.0013 & 1 & -1 \\
\hline
\end{tabular}

Table 3: Wilcoxon Signed Rank Test data

\section{Conclusion}

In this paper, a new approach of a consensus reaching process based on the T1OWA operator is proposed to deal with GDM problems in multi-granular linguistic contexts. Unlike previous consensus models that require balanced linguistic term sets, the new consensus reaching model allows the direct processing of the membership functions of the fuzzy sets modelling the linguistic information and therefore makes the unification process step currently used in previous models unnecessary. Furthermore, because the membership functions are nor required to fulfil extra conditions regarding their balanced or unbalanced distribution within the underlying domain of the variable used to measure preferences, nor they are required to be of the same shape type, the proposed methodology offers a greater degree of flexibility or generality in its application than existing models do.

A comparative study between the T1OWA based consensus model and the unification based consensus model is included using six randomly generated GDM problems with balanced multi-granular information. Under the same conditions of application, i.e. when the distance between fuzzy sets in the T1OWA based approach is defined as the corresponding distance between their centroids significant differences are found between both consensus approaches. Arguably, the T1OWA methodology can be used with guarantee in consensus reaching multigranular linguistic decision making problems.

\section{Appendix: Multi-granular unification based methodology}

In [6], Herrera et al. propose a methodology to deal with multi-granular GDM problems that is based on the application of a unification process by which experts' preferences are transformed into a single domain called basic linguistic term set (BLTS), $S_{T}$. The unification based consensus support system (CSS) model is illustrated in Figure 7, 


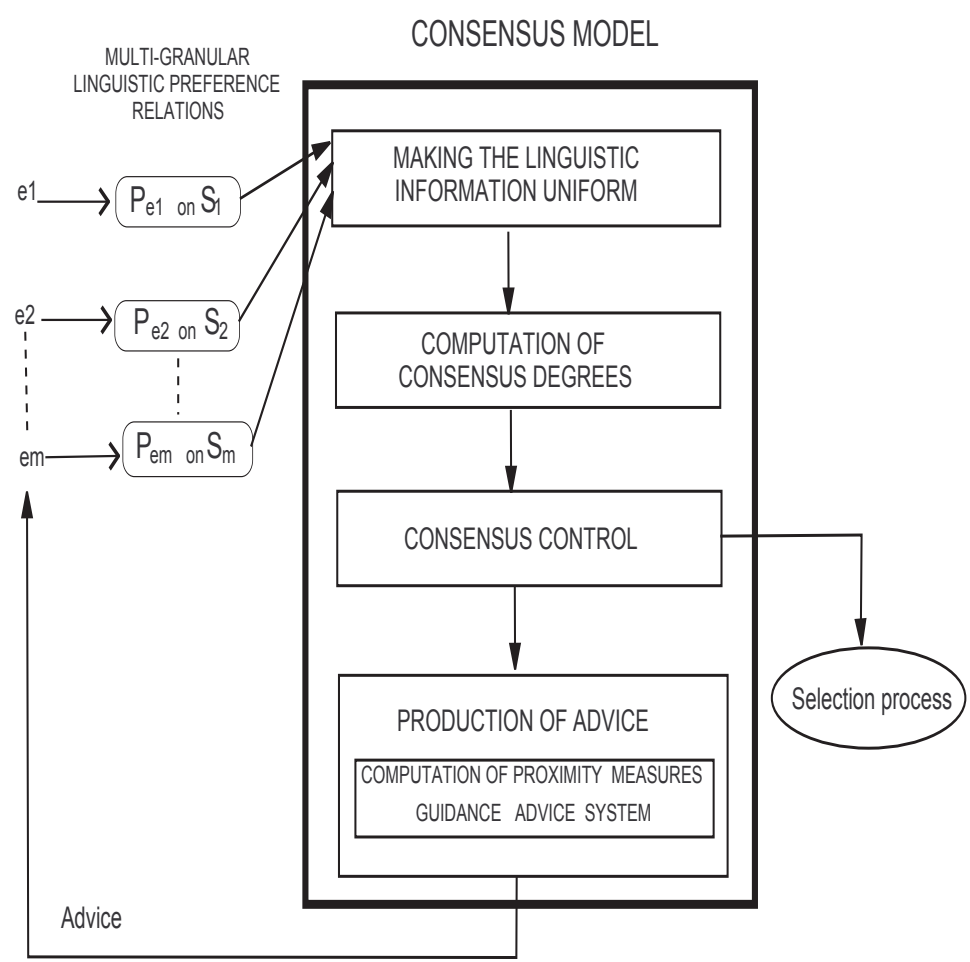

Figure 7: A multi-granular linguistic unification based CSS model

Once $S_{T}$ has been selected, the following multi-granular transformation function is applied to model every linguistic value as a fuzzy set defined on $S_{T}$ :

Definition 7 (Unification transformation [6]). Given two linguistic term sets $S=\left\{l_{0}, \ldots, l_{p}\right\}$ and $S_{T}=\left\{c_{0}, \ldots, c_{g}\right\}$, with $g \geq p$, and $F\left(S_{T}\right)$ the set of fuzzy sets defined on $S_{T}$, the following multi-granular transformation function $\tau_{S S_{T}}: S \rightarrow F\left(S_{T}\right)$,

$$
\tau_{S S_{T}}\left(l_{i}\right)=\left\{\left(c_{h}, \alpha_{h}\right) \mid \alpha_{h}=\max _{y} \min \left\{\mu_{l_{i}}(y), \mu_{c_{h}}(y)\right\}, h=0, \ldots, g\right\}
$$

transforms each element of $S$ into a fuzzy set on $S_{T} ; \mu_{l_{i}}(y)$ and $\mu_{c_{h}}(y)$ are the membership functions of the fuzzy sets associated to the linguistic terms $l_{i}$ and $c_{h}$, respectively.

The following example illustrates the application of the above unification transformation function:

Example 8. Let $S=\left\{l_{0}, l_{1}, l_{2}, l_{3}, l_{4}\right\}$ and $S_{T}=\left\{c_{0}, c_{1}, c_{2}, c_{3}, c_{4}, c_{5}, c_{6}\right\}$ be two term sets represented with the following TFNs

$$
\begin{array}{ll}
l_{0}=(0,0,0.25) & c_{0}=(0,0,0.16) \\
l_{1}=(0,0.25,0.5) & c_{1}=(0,0.16,0.34) \\
l_{2}=(0.25,0.5,0.75) & c_{2}=(0.16,0.34,0.5) \\
l_{3}=(0.5,0.75,1) & c_{3}=(0.34,0.5,0.66) \\
l_{4}=(0.75,1,1) & c_{4}=(0.5,0.66,0.84) \\
& c_{5}=(0.66,0.84,1) \\
& c_{6}=(0.84,1,1)
\end{array}
$$


The fuzzy set obtained when applying $\tau_{S S_{T}}$ to $l_{1}$ is

$$
\tau_{S S_{T}}\left(l_{1}\right)=\left\{\left(c_{0}, 0.39\right),\left(c_{1}, 0.85\right),\left(c_{2}, 0.85\right),\left(c_{3}, 0.39\right),\left(c_{4}, 0\right),\left(c_{5}, 0\right),\left(c_{6}, 0\right)\right\} .
$$

This is depicted in Figure 8:
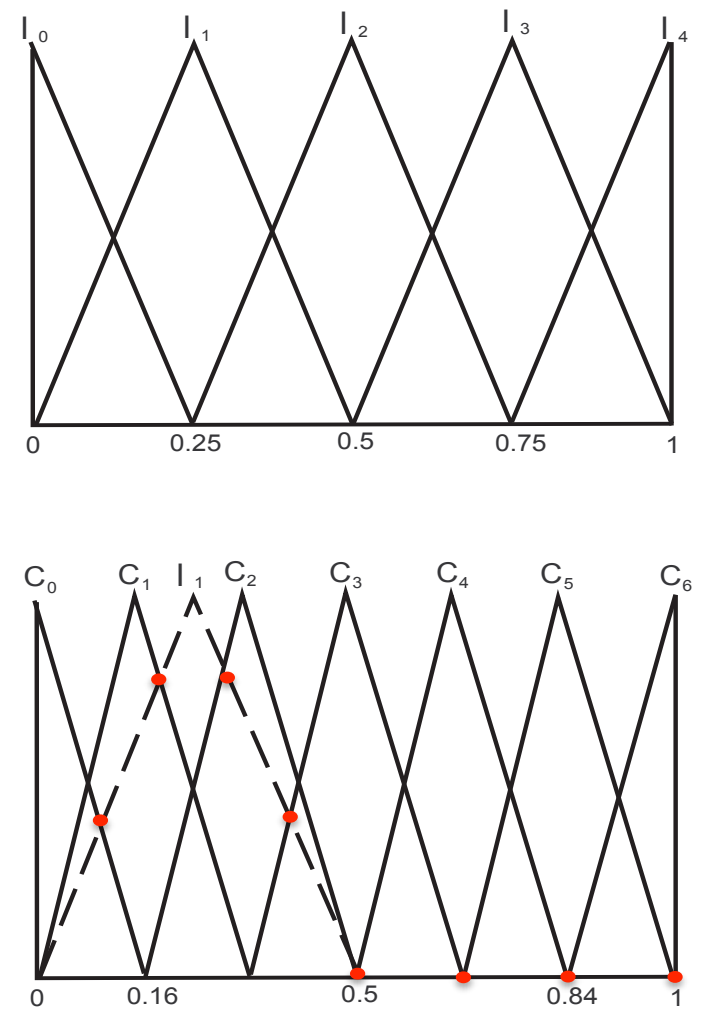

Figure 8: Transforming $l_{1} \in S$ into a fuzzy set on $S_{T}$

\section{Acknowledgements}

This work has been supported by the Research Projects UJA2009/12/25, UJA2011/14/01 and TIN2009-08286.

\section{References}

[1] L. Zadeh, The concept of a linguistic variable and its applications to approximate reasoning, Information Sciences, Part I, II, III 8,8,9 (1975) 199-249,301-357,43-80.

[2] P. P. Bonissone, K. S. Decker, Selecting uncertainty calculi and granularity: An experiment in trading-off precision and complexity, Mach Intell Pattern Recognit 4 (1986) 217-247.

[3] R. de Andrés, J. García-Lapresta, L. Martínez, A multi-granular linguistic model for management decision-making in performance appraisal, Soft Computing 14 (2009) 2134.

[4] M. Espinilla, J. Liu, L. Martínez, An extended hierarchical linguistic model for decisionmaking problems, Computational Intelligence 27 (2011) 489-512. 
[5] F. Herrera, E. Herrera-Viedma, L. Martínez, A fuzzy linguistic methodology to deal with unbalanced linguistic term sets, IEEE Transactions on Fuzzy Systems 16 (2008) 354-370.

[6] F. Herrera, E. Herrera-Viedma, L. Martínez, A fusion aproach for managing multigranularity linguistic term sets in decision making, Fuzzy Sets and Systems 114 (2000) $43-58$.

[7] E. Herrera-Viedma, O. Cordón, M.Luque, A. López, A. Muñoz, A model of fuzzy linguistic irs based on multi-granular linguistic information, International Journal of Approximate Reasoning 34 (2003) 221-239.

[8] E. Herrera-Viedma, L. Martínez, F. Mata, F. Chiclana, A consensus support system model for group decision-making problems with multi-granular linguistic preference relations, IEEE Transactions on Fuzzy Systems 13 (2005) 644-658.

[9] F. J. Cabrerizo, I. J. Pérez, E. Herrera-Viedma, Managing the consensus in group decision making in an unbalanced fuzzy linguistic context with incomplete information, Knowledge-Based Systems, 23 (2010) 169-181.

[10] F. Herrera, E. Herrera-Viedma, J. Verdegay, A sequential selection process in group decision making with linguistic assessment, Information Sciences 85 (1995) 223-239.

[11] F. Herrera, E. Herrera-Viedma, J. Verdegay, Direct approach processes in group decision making using linguistic OWA operators, Fuzzy Sets and Systems 79 (1996) 175-190.

[12] Y. Jiang, Z. Fan, J. Ma, A method for group decision making with multi-granularity linguistic assessment information, Information Sciences 178 (2008) 1098-1109.

[13] S. Zhou, F. Chiclana, R. I. John, J. M. Garibaldi, Type-1 OWA operators for aggregating uncertain information with uncertain weights induced by type-2 linguistic quantifiers, Fuzzy Sets and Systems 159 (2008) 3281-3296.

[14] R. Yager, On ordered weighted averaging aggregation operators in multicriteria decision making, IEEE Transactions on Systems, Man and Cybernetics 18 (1988) 183-190.

[15] S. Zhou, F. Chiclana, R. I. John, J. M. Garibaldi, Alpha-level aggregation: A practical approach to type-1 OWA operation for aggregating uncertain information with applications to breast cancer treatments, IEEE Transactions on Knowledge and Data Engineering 23 (2011) 1455-1468.

[16] F. Chiclana, S.-M. Zhou, The type-1 owa operator and the centroid of type-2 fuzzy sets, in: EUSFLAT-LFA 2011 Proceedings, pp. 15-20.

[17] F. Chiclana, S.-M. Zhou, Type-reduction of general type-2 fuzzy sets: The type-1 owa approach, International Journal of Intelligent Systems 28 (2013) 505-522.

[18] F. Mata, F. Chiclana, S.-M. Zhou, Type-1 owa based multi-granular consensus model, in: ESTYLF 2012, Valladolid (Spain), pp. 235-240.

[19] F. Herrera, E. Herrera-Viedma, J. Verdegay, A linguistic decision process in group decision making, Group Decision and Negotiation 5 (1996) 165-176.

[20] L. Thompson, Negotiation Theory and Research, Psychology Press, 2006. 
[21] E. Herrera-Viedma, F. Herrera, F. Chiclana, A consensus model for multiperson decision making with different preference structures, IEEE Transactions on Systems, Man and Cybernetics-Part A: Systems and Humans 32 (2002) 394-402.

[22] E. Herrera-Viedma, S. Alonso, F. Chiclana, F. Herrera, A consensus model for group decision making with incomplete fuzzy preference relations, IEEE Transactions on Fuzzy Systems 15 (2007) 863-877.

[23] F. Chiclana, J. Tapia-Garcia, M. J. del Moral, E. Herrera-Viedma, A statistical comparative study of different similarity measures of consensus in group decision making, Information Sciences 221 (2013) 110-123.

[24] Y. Dong, Y. Xu, H. Li, B. Feng, The OWA-based consensus operator under linguistic representation models using position indexes, European Journal of Operational Research 203 (2010) 455-463.

[25] G. Zhang, Y. Dong, Y. Xu, H. Li, Minimum-cost consensus models under aggregation operators, IEEE Transactions on Systems, Man, and Cybernetics Part A:Systems and Humans 41 (2011) 1253-1261.

[26] F. Herrera, E. Herrera-Viedma, S. Alonso, F. Chiclana, Computing with words in decision making: Foundations, trends and prospects, Fuzzy Optimization and Decision Making 8 (2009) 337-364.

[27] T. Tanino, Fuzzy preference orderings in group decision making, Fuzzy Sets and Systems 12 (1984) 117-131.

[28] T. Tanino, On Group Decision Making Under Fuzzy Preferences, Multiperson Decision Making Using Fuzzy Sets and Possibility Theory, Kluwer Academic Publishers, pp. 172185.

[29] F. Chiclana, F. Herrera, E. Herrera-Viedma, Integrating three representation models in fuzzy multipurpose decision making based on fuzzy preference relations, Fuzzy Sets and Systems 97 (1998) 33-48.

[30] F. Chiclana, F. Mata, L. Martínez, E. Herrera-Viedma, S. Alonso, Integration of a consistency control module within a consensus model, International Journal of Uncertainty, Fuzziness and Knowledge-Based Systems 16 (2008) 35-53.

[31] I. Millet, The effectiveness of alternative preference elicitation methods in the analytic hierarchy process, Journal of Multi-Criteria Decision Analysis 6 (1997) 41-51.

[32] F. Herrera, E. Herrera-Viedma, J. Verdegay, A model of consensus in group decision making under linguistic assessments, Fuzzy Sets and Systems 79 (1996) 73-87.

[33] F. Herrera, L. Martínez, A model based on linguistic 2-tuples for dealing with multigranularity hierarchical linguistic contexts in multiexpert decision-making, IEEE Transactions on Systems, Man and Cybernetics. Part B: Cybernetics 31 (2001) 227-234.

[34] S. Wang, Applying 2-tuple multigranularity linguistic variables to determine the supply performance in dynamic environment based on product-oriented strategy, IEEE Transactions on Fuzzy Systems 16 (2008) 29-39. 
[35] G. Miller, The magical number seven plus or minus two: Some limits on our capacity of processing information, Psychological Review 63 (1956) 81-97.

[36] S. Saint, J. R. Lawson, Rules for Reaching Consensus. A Modern Approach to Decision Making, Jossey-Bass, San Francisco, 1994.

[37] J. Kacprzyk, M. Fedrizzi, A "soft" measure of consensus in the setting of partial (fuzzy) preferences, European Journal of Operational Research 34 (1988) 316-325.

[38] F. Mata, L. Martínez, E. Herrera-Viedma, An adaptive consensus support system model for group decision-making problems in a multigranular fuzzy linguistic context, IEEE Transactions on Fuzzy Systems 17 (2009) 279-290.

[39] Z. Xu, An automatic approach to reaching consensus in multiple attribute group decision making, Computers and Industrial Engineering 56 (2009) 1369-1374.

[40] J. García-Lapresta, Favoring consensus and penalizing disagreement in group decision making, Journal of Advanced Computational Intelligence and Intelligent Informatics 12 (2008) 416-421.

[41] Z. Wu, J. Xu, Consensus reaching models of linguistic preference relations based on distance functions, Soft Computing 16 (2012) 577-589.

[42] J. M. Tapia García, M. J. Del Moral, M. A. Martínez, E. Herrera-Viedma, A consensus model for group decision making problems with linguistic interval fuzzy preference relations, Expert Systems with Applications 39 (2012) 10022-10030.

[43] L. Susskind, S. McKearnan, J. Thomas-Larmer, The Consensus Building Handbook: A comprehensive guide to reaching agreement, SAGE Publications, 1999.

[44] J. Xu, Z. Wu, A discrete consensus support model for multiple attribute group decision making, Knowledge-Based Systems 24 (2011) 1196 - 1202.

[45] M. M. Deza, E. Deza, Encyclopedia of Distances, Springer Berlin Heidelberg, 2009.

[46] D. Dubois, H. Prade, Fuzzy Sets and Systems: Theory and Applications, Kluwer Academic, New York, 1980.

[47] C. Chakraborty, D. Chakraborty, A theoretical development on a fuzzy distance measure for fuzzy numbers, Mathematical and Computer Modelling 43 (2006) 254-261.

[48] E. Szmidt, J. Kacprzyk, Distances between intuitionistic fuzzy sets, Fuzzy Sets and Systems 114 (2000) 505-518.

[49] R. R. Yager, Owa aggregation over a continuous interval argument with applications to decision making, Systems, Man, and Cybernetics, Part B: Cybernetics, IEEE Transactions on 34 (2004) 1952-1963.

[50] C. Cheng, A new approach for ranking fuzzy numbers by distance method, Fuzzy Sets and Systems 95 (1998) 307-317.

[51] B. Shieh, An approach to centroids of fuzzy numbers, International journal of Fuzzy Systems 9 (2007) 51-54. 
[52] R. Zwick, E. Carlstein, D. V. Budescu, Measures of similarity between fuzzy concepts: a comparative analysis, International Journal of Aproximate Reasoning 1 (1987) 221-242.

[53] S. Alonso, F. Chiclana, F. Herrera, E. Herrera-Viedma, A learning procedure to estimate missing values in fuzzy preference relations based on additive consistency, Lecture Notes Artificial Intelligence 3131 (2004) 227-238.

[54] S. Greenfield, F. Chiclana, S. Coupland, R. I. John, The collapsing method of defuzzification for discretised interval type-2 fuzzy sets, Information Sciences 179 (2009) 20552069.

[55] S. Greenfield, F. Chiclana, S. Coupland, R. I. John, The sampling method of defuzzification for type-2 fuzzy sets: experimental evaluation, Information Sciences 189 (2012) 77-92.

[56] R. D. C. Team, R: A language and environment for statistical computing, R Foundation for Statistical Computing, Vienna, Austria, 2006.

[57] D. C. Montgomery, G. C. Runger, Applied statistics and probability for engineers, Wiley, 2007. 